\title{
Correction to: Associations between change in labour market policies and work stressors: a comparative longitudinal survey data analysis from 27 European countries
}

T. Lunau ${ }^{1 *}$, M. Wahrendorf ${ }^{1}$, N. Dragano ${ }^{1}$, J. Siegrist ${ }^{2}$, K. A. van der Wel ${ }^{3}$ and M. Rigó ${ }^{1}$

Correction to: BMC Public Health 20, 1377 (2020)

https://doi.org/10.1186/s12889-020-09364-3

It was highlighted that in the original article [1] there was a misalignment in Tables 3 and 5. This Correction article shows the correct Tables 3 and 5 . The original article has been updated.

The original article can be found online at https://doi.org/10.1186/s12889-02 0-09364-3

* Correspondence: Thorsten.Lunau@uni-duesseldorf.de

'Institute of Medical Sociology, Centre for Health and Society, Medical

Faculty of the University of Düsseldorf, Düsseldorf, Germany

Full list of author information is available at the end of the article

(c) The Author(s). 2020 Open Access This article is licensed under a Creative Commons Attribution 4.0 International License, which permits use, sharing, adaptation, distribution and reproduction in any medium or format, as long as you give appropriate credit to the original author(s) and the source, provide a link to the Creative Commons licence, and indicate if changes were made. The images or other third party material in this article are included in the article's Creative Commons licence, unless indicated otherwise in a credit line to the material. If material is not included in the article's Creative Commons licence and your intended use is not permitted by statutory regulation or exceeds the permitted use, you will need to obtain permission directly from the copyright holder. To view a copy of this licence, visit http://creativecommons.org/licenses/by/4.0/. The Creative Commons Public Domain Dedication waiver (http://creativecommons.org/publicdomain/zero/1.0/) applies to the data made available in this article, unless otherwise stated in a credit line to the data. 
Table 3 Association between individual variables and work stressors (based on linear multilevel models)

\begin{tabular}{|c|c|c|c|c|c|c|c|c|c|c|c|}
\hline & & \multicolumn{2}{|l|}{ ERI } & \multicolumn{2}{|l|}{ Effort } & \multicolumn{2}{|l|}{ Reward } & \multicolumn{2}{|c|}{ Job strain } & \multicolumn{2}{|l|}{ Control } \\
\hline & & b & $p$ & b & $p$ & b & $\mathrm{p}$ & b & $p$ & b & $\mathrm{p}$ \\
\hline \multicolumn{12}{|l|}{ Model 0} \\
\hline \multirow[t]{3}{*}{ Variance } & Level 3 (Country) & .0007 & & .0032 & & 0.0019 & & .0017 & & .0046 & \\
\hline & Level 2 (Country-years) & .0004 & & .0004 & & 0.0008 & & .0004 & & .0005 & \\
\hline & Level 1 (Individual) & .0326 & & .0449 & & 0.0312 & & .0418 & & .0479 & \\
\hline \multicolumn{12}{|l|}{ Model 1} \\
\hline \multicolumn{12}{|l|}{ Level 1 (Individuals) } \\
\hline \multirow[t]{2}{*}{ Year (ref. 2005) } & 2010 & -.0001 & .992 & -.0113 & .072 & -.0131 & .039 & .0007 & .914 & -.0156 & .013 \\
\hline & 2015 & -.0107 & .055 & -.0030 & .938 & .0215 & .001 & .0064 & .313 & -.0157 & .013 \\
\hline \multirow[t]{2}{*}{ Age (ref. $<=30$ ) } & $30<$ age $<55$ & .0063 & $\leq .001$ & -.0096 & $\leq .001$ & -.0242 & $\leq .001$ & -.0193 & $\leq .001$ & .0229 & $\leq .001$ \\
\hline & age $>=55$ & -.0087 & $\leq .001$ & -.0447 & $\leq .001$ & -.0378 & $\leq .001$ & -.0433 & $\leq .001$ & .0233 & $\leq .001$ \\
\hline Gender (ref. male) & female & .0199 & $\leq .001$ & .0090 & $\leq .001$ & -.0244 & $\leq .001$ & .0338 & $\leq .001$ & -.0461 & $\leq .001$ \\
\hline \multirow[t]{3}{*}{ ISCO (ref. ISCO1) } & ISCO2 & -.0020 & .445 & .0319 & $\leq .001$ & .0357 & $\leq .001$ & -.0253 & $\leq .001$ & .0681 & $\leq .001$ \\
\hline & ISCO3 & -.0275 & $\leq .001$ & .0163 & $\leq .001$ & .0638 & $\leq .001$ & -.0741 & $\leq .001$ & .1328 & $\leq .001$ \\
\hline & ISCO4 & -.0406 & $\leq .001$ & .0353 & $\leq .001$ & .1100 & $\leq .001$ & -.1248 & $\leq .001$ & .2445 & $\leq .001$ \\
\hline \multirow[t]{4}{*}{ Contract (ref. permanent contract) } & fixed term contract & .0318 & $\leq .001$ & -.0120 & $\leq .001$ & -.0652 & $\leq .001$ & .0215 & $\leq .001$ & -.0404 & $\leq .001$ \\
\hline & temporary employment & .0615 & $\leq .001$ & -.0064 & .364 & -.1082 & $\leq .001$ & .0625 & $\leq .001$ & -.0914 & $\leq .001$ \\
\hline & apprenticeship & -.0357 & $\leq .001$ & -.0373 & $\leq .001$ & .0230 & .010 & -.0214 & .024 & -.0232 & .017 \\
\hline & other & .0170 & $\leq .001$ & -.0295 & $\leq .001$ & -.0595 & $\leq .001$ & -.0118 & $\leq .001$ & -.0111 & .001 \\
\hline \multirow[t]{4}{*}{ NACE (ref. agriculture) } & industry & .0393 & $\leq .001$ & .0633 & $\leq .001$ & .0007 & .880 & .0543 & $\leq .001$ & -.0209 & $\leq .001$ \\
\hline & services & .0296 & $\leq .001$ & .0409 & $\leq .001$ & -.0076 & .112 & .0365 & $\leq .001$ & -.0229 & $\leq .001$ \\
\hline & public administration & -.0116 & .036 & .0123 & .058 & .0328 & $\leq .001$ & -.0144 & .016 & .0262 & $\leq .001$ \\
\hline & other services & -.0043 & .402 & -.0022 & .719 & .0021 & .662 & -.0126 & .023 & .0032 & .509 \\
\hline Constant & & .7996 & $\leq .001$ & 1.3890 & $\leq .001$ & 1.7494 & $\leq .001$ & .8127 & $\leq .001$ & 1.7326 & $\leq .001$ \\
\hline \multirow[t]{3}{*}{ Variance } & Level 3 (Country) & .0007 & & .0035 & & .0017 & & .0017 & & .0032 & \\
\hline & Level 2 (Country years) & .0003 & & .0004 & & .0005 & & .0004 & & .0004 & \\
\hline & Level 1 (Individual) & .0317 & & .0439 & & .0289 & & .0385 & & .0404 & \\
\hline N & & 64,659 & & 64,659 & & 64,659 & & 67,114 & & 67,114 & \\
\hline
\end{tabular}

Note: Model 0 presents the variance of the outcome variable on the individual (level 1), the country year (level 2) and the country level (level 3) 


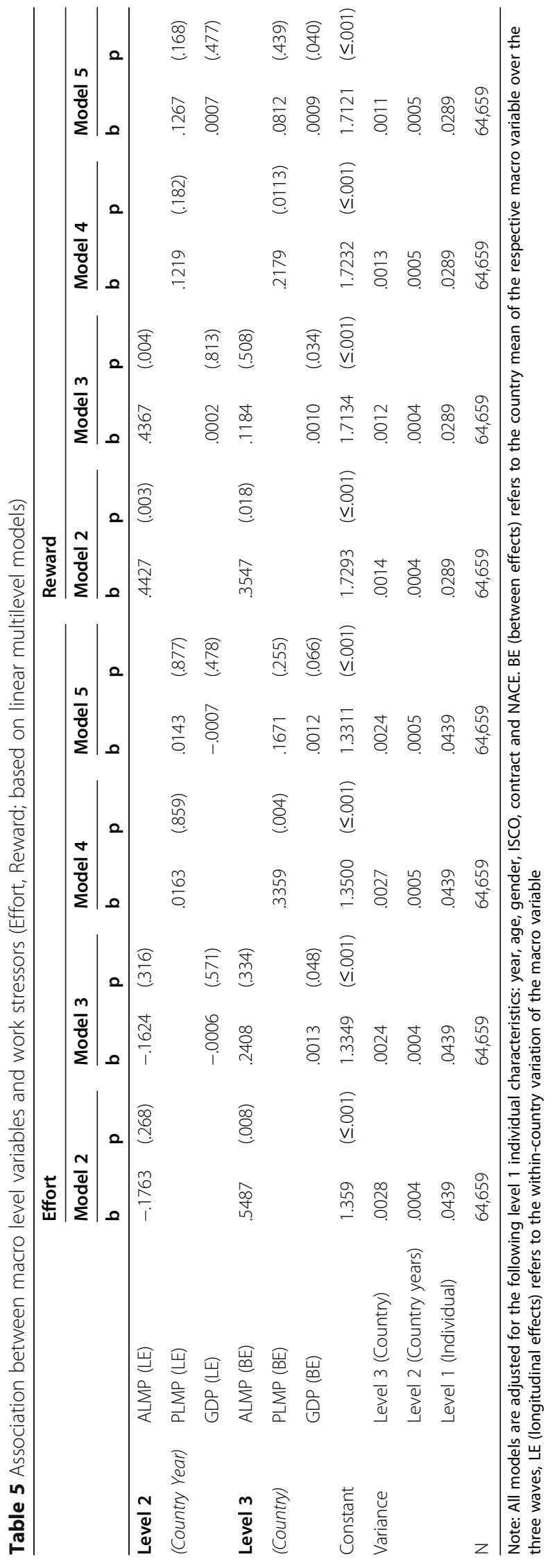




\section{Author details}

${ }^{1}$ Institute of Medical Sociology, Centre for Health and Society, Medical

Faculty of the University of Düsseldorf, Düsseldorf, Germany. ${ }^{2}$ Senior

professorship on work stress research, Medical Faculty, University of

Düsseldorf, Düsseldorf, Germany. ${ }^{3}$ Department of Social Work, Child Welfare and Social Policy, Oslo Metropolitan University, Oslo, Norway.

Published online: 19 October 2020

\section{Reference}

1. Lunau, et al. Associations between change in labour market policies and work stressors: a comparative longitudinal survey data analysis from 27 European countries. BMC Public Health. 2020;20:1377. https://doi.org/10. 1186/s12889-020-09364-3. 